\title{
La Población Indígena Y El Cambio Climático En Centroamérica: Aproximaciones a Su Impacto
}

\author{
Camacho, María Fernanda Morales ${ }^{1}$ \\ Soto- Acosta, Willy²
}

\section{Resumo}

Nos últimos anos, popularizou-se a expressão migrantes ambientais, apesar de o conceito não ter sido, ainda, introduzido, na doutrina do direito internacional e, tampouco, ter havido o reconhecimento da categoria refugiado ambiental. Apesar destas lacunas, as alterações climáticas têm tido um impacto sobre as decisões, de povos mais vulneráveis, tanto para empreender uma migração interna quanto externa. Concretamente, a população indígena centro-americana representa um grupo de grande vulnerabilidade, dadas as condições sócio-econômicas e pelo fato de estarem, as comunidades, assentadas em áreas de grande risco no que diz respeitos a desastres naturais. Desta forma, eventos climáticos, tais como: furações, tormentas e secas contribuem para a exclusão destas minorias na região.

Indígenas. América Central. Alterações climáticas. Migração. Deslocamentos. Migrantes ambientales

\section{Abstracto}

En los últimos años se ha popularizado el términos de migrantes ambientales, a pesar de que el concepto aún no se ha introducido en la doctrina del derecho internacional menos aún, el reconocimiento de categorías como refugiado ambiental. No obstante estos vacíos, el cambio climático tiene un impacto sobre las decisiones de las poblaciones más vulnerables para desplazarse a nivel interno y externo. Concretamente, la población indígena en Centroamérica representa un grupo de gran vulnerabilidad debido a sus condiciones socioeconómicas y la ubicación de sus asentamientos en áreas de gran riesgo ante los fenómenos naturales. De esta forma, eventos climatológicos como los huracanes, tormentas y sequías contribuyen con la perpetuación y exclusión de estas minorías en la región.

Indígenas. Centroamérica. Cambio climático. Migración. Desplazamientos. Migrantes ambientales

\footnotetext{
1 Licenciada en Relaciones Internacionales con énfasis en comercio internacional de la Universidad Nacional (UNA). Investigadora en la Secretaría General de FLACSO. Correo: mfmcamacho@gmail.com

2 Sociólogo y Politólogo. Profesor de la Escuela de Relaciones Internacionales de la Universidad Nacional (UNA). Correo: altivohaciaadelante@gmail.com
} 


\section{Introducción}

Junto con las migraciones tradicionales originadas por las condiciones socioeconómicas y de seguridad en Centroamérica, constituyéndola como una región de tránsito y expulsora de migrantes, se une el elemento ambiental como un factor expulsor. Esto porque conduce a que las poblaciones más vulnerables a condiciones de inseguridad en materia de acceso a los bienes públicos como el agua, experimenten dificultades para asegurar necesidades tan básicas como la alimentación y posibilidades de crecimiento por productividad que van más allá de la subsistencia.

Las poblaciones indígenas centroamericanas han vivido históricas condiciones de marginalidad, las cuales se acentúan por el impacto de fenómenos naturales que contribuyen con la profundización de la pobreza y la exclusión. El presente artículo se propone analizar el impacto del cambio climático en esos grupos de la región centroamericana. Para ello se realizará una aproximación al estado actual de esa población en Centroamérica posteriormente, un acercamiento a las migraciones tradicionales y ambientales y, finalmente, un análisis sobre la relación del cambio climático, las migraciones y las poblaciones indígenas.

\section{El panorama de las poblaciones indígenas en Centroamérica}

En el marco de este breve acercamiento a las condiciones socio-demográficas de los pueblos indígenas centroamericanos, resulta necesario estar consciente de la existencia histórica de considerables divergencias a la hora de comparar cálculos y estimaciones de población indígena en la región, e inclusive, de la ausencia frecuente de información sobre la materia. Es hasta la década de 2000 que la realización de censos permite una mejor comparación de los datos estadísticos, aunque aún con diferencias considerables entre las cifras oficiales y las provenientes de organizaciones indígenas u organismos internacionales.

Así el panorama, el número total de indígenas en la región responde más bien a una estimación que, para 2008 y según cifras del Instituto Interamericano de Derechos 
Humanos, era de unos 7,7 millones de personas, representando un $18 \%$ de la población total (IIDH en Estado de la Región 2011 362). Con el fin de partir de una estandarización de fuentes que brinde una referencia comparable, la tabla a continuación muestra las cifras oficiales de población indígena en América Central según los censos nacionales más recientes.

Tabla 1: Población Indígena en América Central Según Censos Nacionales

(Fuente: Elaboración propia a partir de censos nacionales)

\begin{tabular}{|c|c|c|c|c|c|}
\hline \multirow[b]{2}{*}{ PAÍS } & \multirow[b]{2}{*}{ GRUPOS ÉTNICOS } & \multicolumn{3}{|c|}{ RESULTADOS CENSALES } & \multirow{2}{*}{ Fuente- Ano } \\
\hline & & $\begin{array}{l}\text { Población } \\
\text { indígena }\end{array}$ & $\begin{array}{l}\text { Población } \\
\text { total }\end{array}$ & $\begin{array}{l}\text { Población } \\
\text { indígena }\end{array}$ & \\
\hline Belice & $\begin{array}{l}\text { Garífuna, Maya q'eqchí, Maya } \\
\text { nopán, Maya yucateco. }\end{array}$ & 30107 & 312.698 & $9,6 \%$ & $\begin{array}{l}\text { Statistical } \\
\text { Institute of } \\
\text { Belize.2010 }\end{array}$ \\
\hline Costa Rica & $\begin{array}{c}\text { Bribí, Brunca o Boruca, Cabécar, } \\
\text { Chorotega, Huetar, Maleku o } \\
\text { Guatuso, Nögbe o Guaymí, Terire } \\
\text { o Térraba. }\end{array}$ & 104143 & 4.301 .712 & $2,4 \%$ & $\begin{array}{l}\text { Instituto Nacional } \\
\text { de Estadística y } \\
\text { Censos.2011 }\end{array}$ \\
\hline EI Salvador & $\begin{array}{l}\text { Lenca, Kakawira (Cacaopera), } \\
\text { Nahua-Pipil. }\end{array}$ & 291.461 & 5.744 .113 & $5,1 \%$ & $\begin{array}{l}\text { Dirección } \\
\text { General de } \\
\text { Estadística y } \\
\text { Censos.2007 }\end{array}$ \\
\hline Guatemala & $\begin{array}{c}\text { Maya (incluyendo los } 21 \text { grupos } \\
\text { mayas existentes), Xinca, } \\
\text { Garífuna, Ladino. }\end{array}$ & 4610440 & 11.237.196 & $41 \%$ & $\begin{array}{l}\text { Instituto Nacional } \\
\text { de Estadística. } \\
2002\end{array}$ \\
\hline Honduras & $\begin{array}{c}\text { Miskitu, Lenca, Tolupan, Tawahka, } \\
\text { Maya Chortis, Nahoa, Pech, } \\
\text { Garifuna. }\end{array}$ & 496000 & 7621000 & $6,5 \%$ & $\begin{array}{l}\text { Instituto Nacional } \\
\text { de Estadística, a } \\
\text { través de } \\
\text { KIVLAK/GIZ.2001 }\end{array}$ \\
\hline Nicaragua & $\begin{array}{l}\text { Miskitu, Mestizos de la Costa } \\
\text { Caribe, Chorotega Nahua-Mange, } \\
\text { Creole (kriol) Xiu-Sutiava, } \\
\text { Cacaopera-Matagalpa, Nahoa- } \\
\text { Nicarao, Mayangna- Sumu. }\end{array}$ & 443847 & 5142098 & $8.6 \%$ & $\begin{array}{l}\text { Instituto Nacional } \\
\text { de Información } \\
\text { para el } \\
\text { Desarrollo.2005 }\end{array}$ \\
\hline Panamá & $\begin{array}{c}\text { Kuna, Ngäbe,Buglé, Emberá, } \\
\text { Wounaan, Teribe/Naso, Bokota y } \\
\text { Bri Bri. }\end{array}$ & 417559 & $3,405,813$ & $12,3 \%$ & $\begin{array}{l}\text { Instituto Nacional } \\
\text { de Estadística y } \\
\text { Censo } 2010\end{array}$ \\
\hline
\end{tabular}

A pesar de utilizar fuentes homologables, debido a las diferencias en las fechas de los censos, realizar comparaciones acarrea limitantes, aunque permite tener una perspectiva general del panorama desde la información oficial. Vale resaltar que, en 
casos como el de Guatemala, el peso demográfico de los pueblos indígenas es tal, que "técnicamente hablando, dejan de ser minorías étnicas y pasan a ser mayorías discriminadas" (BARIÉ 2003 p. 37). Diferente es en países como Costa Rica, en donde las cifras arrojan un porcentaje de población indígena que no alcanza, para este cálculo censal, el 3\% de la población total.

Ahora bien, tanto en los casos en los que el peso demográfico es significativo, como en los que el porcentaje es proporcionalmente mucho menor, los pueblos indígenas comparten condiciones de vulnerabilidad que son producto de las dinámicas históricas de exclusión, y que evidencian una profunda desigualdad. Basta con acercarse a algunas de las alarmantes cifras del Foro Permanente sobre Asuntos Indígenas de la Organización de las Naciones Unidas -publicadas en 2010-, para visualizar que en Panamá los índices de pobreza son 5.9 mayores para población indígena que para población no indígena que en Guatemala, más del $50 \%$ de los jóvenes indígenas entre los 15 y los 19 años no completaron la educación primaria, en comparación con aproximadamente un tercio de los jóvenes no indígenas que en Honduras, aproximadamente un 95\% de los niños indígenas menores de 14 años sufren desnutrición (ONU 2010, p. 14).

\section{En Palabras del Estado de la Región:}

"estos grupos enfrentan barreras que limitan sus oportunidades y el desarrollo de sus capacidades. Su rezago en el acceso a servicios como salud y educación, agua potable y saneamiento, mercado laboral e infraestructura productiva, queda manifiesto en las brechas en su perfil demográfico con respecto al resto de la población. Más allá de los rezagos en materia demográfica, las poblaciones rurales e indígenas de Centroamérica continúan enfrentando desventajas socioeconómicas que les impiden mejorar sus niveles de desarrollo y calidad de vida. La pobreza, flagelo no superado en la región, las afecta con mayor crudeza”. (2011, p.386).

Estas circunstancias, lo que el Estado de la Región llama "el rezago de la transición demográfica”, Estado de la Región (2011, p.362), se ven aunadas al hecho de que tradicionalmente los grupos indígenas habitan sitios "físicamente aislados, frágiles y 
difíciles, que los hacen especialmente vulnerables" (UICN 2008 4), y a los retos en materia de reconocimiento de sus derechos indígenas. Sobre este último aspecto, cabe resaltar que a la fecha, solo Costa Rica, Guatemala, Honduras y Nicaragua han ratificado el Convenio 169 sobre Pueblos Indígenas y Tribales en Países Independientes de la Organización Internacional del Trabajo, referente en la protección de los derechos colectivos de los pueblos originarios.

Los derechos indígenas territoriales -por citar un caso particularmente pertinente-, no solo no figuran del todo en algunas Constituciones centroamericanas, sino que además, en muchos de los casos en los que sí existe una mención de los temas de tierras y recursos naturales, esta es general y no se refiere en específico a los pueblos indígenas como colectividades.

\section{Los desplazamientos en Centroamérica: migraciones tradicionales y migraciones ambientales en la región Migraciones Tradicionales}

Según Casasfranco (2002, p.78), la dinámica migratoria en la región centroamericana fue más notoria a partir de la década de los ochenta producto de la crisis política en la que estaba sumergida la región.

Antes de esa fecha, los desplazamientos obedecían a procesos de colonización agrícola y se caracterizaban por su dispersión en diferentes regiones a lo interno del país. Incluso, en el contexto de la II Guerra Mundial, Centroamérica fue una región receptora de inmigrantes provenientes de Europa y Asia.

Para el caso de México y de Centroamérica, los desplazamientos hacia la frontera norte no fueron regulares, pues están y estuvieron más vinculados por efectos coyunturales (crisis), demanda temporal en el sector agrícola, y no correspondían a todos los países del continente. Además, los desplazamientos se incrementaron a partir de los periodos de guerra o entreguerras, pero con mayor énfasis en los años ochenta del siglo pasado, Netzahualcoyotzi y Furlong, (2002, p.74). De esta forma, en la década de los ochenta las guerras civiles incidieron en un desplazamiento cercano a los dos millones de personas que provenían de El Salvador, Guatemala y 
Nicaragua y se dirigían a otras zonas de su propio país no afectadas por los enfrentamientos, a países vecinos o a destinos extra-regionales.

Ejemplo de ello fue la oleada migratoria de ciudadanos salvadoreños hacia los Estados Unidos. En el país norteamericano residían cerca de un millón de salvadoreños, muchos de ellos expulsados por la violencia en su tierra natal (ver tabla 1):

Tabla 2: Estimado de población (en miles) residente no autorizada en los Estados Unidos, Top 15 países: 1990-2000 (Fuente: Homeland Security. (2000). Estimates of the Unathorized. Immigrant Population Residing in the United States: 1990 to 2000.)

\begin{tabular}{lrrrrr}
\hline Country of origem & Estimated population & Growth & \multicolumn{2}{r}{ Percent of total pop. } \\
\hline & $\mathbf{2 0 0 0}$ & $\mathbf{1 9 9 0}$ & $\mathbf{1 9 9 0} \mathbf{2 0 0 0}$ & $\mathbf{2 0 0 0}$ & $\mathbf{1 9 9 0}$ \\
& $(1)$ & $(2)$ & $(3)-(1)-(2)$ & $(4)$ & $(5)$ \\
\hline ALL COUNTRIES & 7.000 & 3.500 & 3.500 & $100,0 \%$ & $100,0 \%$ \\
\hline Mexico & 4.808 & 2040 & 2768 & $68,7 \%$ & $58,35 \%$ \\
\hline EI Salvador & 189 & 298 & -101 & $2,7 \%$ & $8,5 \%$ \\
\hline Guatemala & 144 & 118 & 26 & $2,1 \%$ & $3,4 \%$ \\
\hline Colombia & 141 & 51 & 91 & $2,0 \%$ & $1,4 \%$ \\
\hline Honduras & 138 & 42 & 96 & $2,0 \%$ & $1,2 \%$ \\
\hline China & 115 & 70 & 45 & $1,6 \%$ & $2,0 \%$ \\
\hline Equador & 108 & 37 & 71 & $1,5 \%$ & $1,0 \%$ \\
\hline Dominican Republic & 91 & 46 & 45 & $1,3 \%$ & $1,3 \%$ \\
\hline Philippines & 85 & 70 & 14 & $1,2 \%$ & $2,0 \%$ \\
\hline Brazil & 77 & 20 & 58 & $1,1 \%$ & $0,6 \%$ \\
\hline Haiti & 76 & 67 & 8 & $1,1 \%$ & $1,9 \%$ \\
\hline India & 70 & 28 & 41 & $1,0 \%$ & $0,8 \%$ \\
\hline Peru & 61 & 27 & 34 & $0,9 \%$ & $0,8 \%$ \\
\hline Korea & 55 & 24 & 31 & $0,8 \%$ & $0,7 \%$ \\
\hline Canada & 47 & 25 & 22 & $0,7 \%$ & $0,7 \%$ \\
\hline All other ountries & 795 & 537 & 259 & $11,4 \%$ & $15,3 \%$ \\
\hline
\end{tabular}


De acuerdo con la tabla 2, se da un descenso en la población salvadoreña residente ilegal en los Estados Unidos en la década de los noventas respecto al año 2000, producto de las prerrogativas brindadas por el Gobierno estadounidense como el estatus de protección temporal, a principios de los noventas. Ya más tarde, eso se debe a leyes como NACARAi. No obstante, se evidencia una tendencia al alza en la llegada de hondureños y guatemaltecos a los Estados Unidos en condiciones irregulares, con crecimientos relativos de $96 \%$ y $26 \%$, respectivamente (HOMELAND SECURITY, 2000, p.9).

Con el proceso de pacificación en Centroamérica inicia el retorno de migrantes a sus tierras de origen, especialmente de salvadoreños y guatemaltecos. No obstante, la persistencia de condiciones económicas adversas y la creciente vulnerabilidad ante fenómenos ambientales (huracanesii, terremotos, etc.) provoca una nueva oleada de migrantes, los llamados "migrantes ambientales", esta vez huyendo del impacto socioeconómico producido por los fenómenos climáticos.

Surgen entonces dos lógicas de desplazamiento en la región: una al sur, especialmente de nicaragüenses hacia Costa Rica y otra al norte, específicamente ciudadanos de Guatemala, Honduras y el Salvador hacia México, pero teniendo como principal destino los Estados Unidos, en búsqueda del "sueño americano".

Actualmente, y de acuerdo con Where, We're From, para el año 2010 se contabilizaban un total 2664852 migrantes provenientes de Centroamérica en los Estados Unidos.

Dentro de esta cifra sobresale El Salvador con 1116420 migrantes residiendo en este país, seguido por Guatemala con 753720 y Honduras 469 202. Estos tres países representan más del $50 \%$ de los y las centroamericanos en esa nación (ver gráfico 1): 
Gráfico 1: Cantidad de migrantes Centroamericanos (por país de origen) en Estados Unidos (2010) Fuente: Elaboración propia a partir de Organización Internacional para las Migraciones (2014).

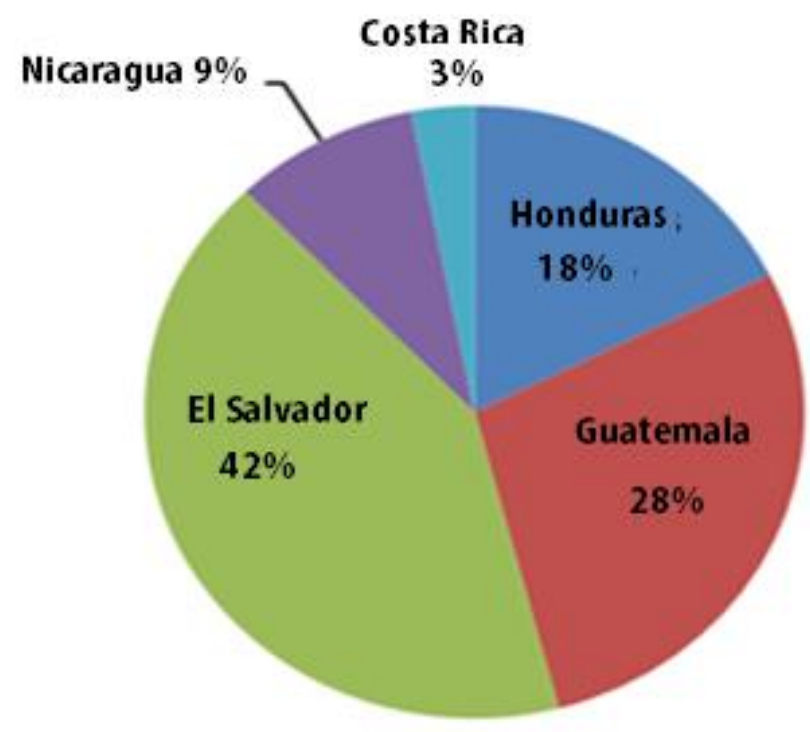

\section{Migraciones ambientales en Centroamérica}

François Gemenne, investigador del Instituto de Desarrollo Sostenible y Relaciones Internacionales, con sede en París, manifestó lo siguiente en la Cumbre del Clima de Poznan, Polonia, realizada en diciembre del 2008:

Claro que ya hay refugiados por motivos ambientales. Hay habitantes de islas muy bajas que se están moviendo por la subida del nivel del mar y la erosión costera, migraciones dentro de China por la desertificación y gente que intenta salir de Bangladesh porque sufren inundaciones cada vez con más frecuencia. Los factores ambientales influyen...

Hemos identificado 22 puntos calientes. Muchos africanos, huyendo de la desertificación, cruzan a Yemen intentando llegar a Arabia Saudí. Por eso Arabia ha levantado un muro con Yemen. O la frontera entre Bangladesh y la India. Bangladesh acusa a India de inundarle con una presa y de sufrir la subida del nivel del mar. India planea levantar una frontera (citado por Méndez, 2018).

Se ha acuñado el concepto de refugiado ambiental para referirse a: 
... las personas, pueblos y, en las situaciones más graves, ciudades que se han visto obligados a trasladarse desde su tierra natal, debido a problemas derivados con el ambiente, como desastres naturales: huracanes o tsunamis, y también por otras razones de devastación como son la deforestación, desertificación, inundaciones, o sequías, con la consecuente falta de agua, alimentos y energía, y riesgo de enfermedades, lo que hace que para estas personas, existan pocas o ninguna esperanza de retorno.

Estas personas son los llamados "desplazados ambientales", término que incluye no sólo a aquellos que tienen que trasladarse a otras zonas dentro de un mismo país, sino también a los que suelen cruzar fronteras internacionales. Al intentar cruzar las fronteras hacia otros territorios más seguros, miles de estos desplazados mueren cada año en las rutas migratorias, por las políticas restrictivas de los países a los que se dirigen y la militarización de las fronteras (BORRÁS, 2008 p.1).

Se pueden distinguir tres tipos de desplazados. Primero, los que tiene que movilizarse temporalmente a raíz eventos extremos tales como terremotos, tsunamis, huracanes, inundaciones pero tiempo después de los acontecimientos pueden regresar a sus lugares de origen. También está un segundo tipo: el que conforman las personas que no pueden regresar a su hábitat debido al grado de destrucción o debido a la explotación de los recursos naturales. Un tercer tipo lo constituyen las personas cuyas sus tierras fueron tomadas (mediante compra y/o expropiación) para darle otro destino económico (por ejemplo, construcción de mega-proyectos turísticos en lo que antes eran poblados de pescadores artesanales) (BORRÁs, 2008, pp.3-4).

En algunos casos la diferencia entre estas situaciones es muy tenue así como difícil establecer si el acto de abandonar el hábitat es voluntario u obligado.

La siguiente tabla identifica algunos factores que generan este tipo de desplazamientos: 
Tabla 3: Causas que propician refugiados ambientales

Fuente: Tomado de Perales, 20105 (Elaborado por Lastiri, Angélica).

NATURALES

Catástrofes

ambientales 0

desastres
Procesos a

largo plazo
ANTROPÓGENICAS

Conflictos

políticos y

militares
Factores

Socioeconómicos
- Inundaciones

- Tifones

- Sequías

- Plagas

- Terremotos

- Maremotos.

- Olas de calor

- Incremento en el nivel del mar

- Erupciones volcánicas

- Tormentas

- Tornados
- Desertificación

- Degradación de

- tierras agrícolas

- Uso excesivo e inadecuado de los recursos hídricos

- Erosión de los suelos, deforestación.

- Vertidos de petróleo o sustancias químicas a ríos o costas.

- Accidentes químicos 0 nucleares.
- Destrucción de Cosechas

- Utilización de armas químicas

- Bombardeos
- Distribución de los recursos.

- Proyectos de Desarrollo

- Escasez de Recursos

La magnitud del problema es alarmante. En efecto, “... para la ONU, el $60 \%$ de los movimientos migratorios son causados por el cambio climático y por los desastres como las sequías o inundaciones. El organismo estima que en 15 años habrá, por lo menos, 50 millones de refugiados por el deterioro del medio ambiente en el mundo, los cuales afectaran, principalmente a los países más pobres" (Cáceres, 2010, p.11). El concepto de migración ambiental, entonces, resulta complejo y polémico en la medida que los desplazamientos de las personas obedecen a múltiples causas. Tal y como lo señala Ferris (2015): "Las personas deciden desplazarse por múltiples razones. Los factores ambientales pueden ser un motivo de peso para decidir 
migrar, pero usualmente se entremezcla con factores económicos, familiares incluso, de tolerancia personal al riesgo." Además, los patrones de migración por casusa ambientales se ven afectado según la severidad del evento y su duración, lo que determinará si se trata de desplazamientos temporales, permanentes, internos o internacionales (FRITZ 2010).

De acuerdo con Fritz (2010), las migraciones ambientales se deben analizar de acuerdo a tres características: la vulnerabilidad ante los fenómenos, la resiliencia para recuperarse de los mismos, y la adaptabilidad para convivir con las condiciones cambiantes producto de las variaciones climáticas. En este contexto, la Organización Mundial para las Migraciones (OIM) estima que en los próximos 40 años un rango comprendido entre los 25 millones y los 1000 millones de personas podrían desplazarse por los efectos del cambio climático, International Organization for Migration (2012).

En el caso centroamericano se debe señalar la vulnerabilidad de la región por su posicionamiento geográfico, lo que la hace propensa a sufrir los embates de fenómenos como las tormentas, sequías o los huracanes. Tal y como lo señalan Arbo y $\operatorname{Ordaz}(2011$, p. 6):

La región conformada por México y Centroamérica es altamente vulnerable a los fenómenos climáticos. Sobre ella se han suscitado diferentes desastres naturales que han traído costos importantes en 1998 el huracán Micth azotó Centroamérica, en 2005 el huracán Stan afectó México y Guatemala la tormenta tropical Noel causó severas inundaciones en Tabasco, en México.

Esto ha situado a los países de esta región dentro de las primeras posiciones de(sic) Índice Global de Riesgo Climático (IGRC). Harmelin señala a Honduras como el país en la tercera posición de IGRC construido para el periodo 1990-2008, Nicaragua en la sexta posición y México en la posición 30.

De igual manera, la CEPAL señala esta condición de riesgo de la región al mencionar que en el periodo 1930-2011 se registraron 291 eventos extremos asociados con fenómenos climáticos, con un crecimiento anual de los desastres en las últimas tres décadas respecto al decenio de 1970 (CEPAL 2012, p.11). 
Así en la región centroamericana (también aplica para el caso mexicano y de África Oriental) se podrían describir movimientos circulares influenciados por el ambiente, en la medida que se realizan a nivel interno, es decir, del campo a la ciudad, donde los y las agricultores migran para compensar la baja productividad agrícola en estas áreas (Fritz 2010).

Tal y como lo señalara el estudio que realizó Mexican Migration Project, sobre los patrones de migración de este país vecino, en el que, de una muestra de 19906 hogares mexicanos, 10086 contaban con terrenos secos no aptos para la agricultura. De esta forma "es posible inferir que el ambiente sí está jugando un papel importante en incentivar la migración de los mexicanos a los Estados unidos", (ARBO Y ORDAZ, 2011, p.7).

Un claro ejemplo del impacto de un fenómeno natural en la estabilidad socioeconómica de la región fue el Huracán Mitch que afectó el istmo en 1998 (RODRÍGUEZ 2013, p.13).

En el año 1998, el Huracán Mitch en Centroamérica y sus efectos, acentuaron las desigualdades socio-económicas en los países región, lo cual agravó los riesgos de las poblaciones más vulnerables y la consecuente incapacidad de los ecosistemas y medios de producción en las zonas afectadas, generando la expulsión de población y grandes dificultades para la generación de empleo local.

Este evento en la región Centroamericana afectó el desafío de lograr la reinserción productiva y el acceso a la tierra de las personas que regresaron a sus países de origen luego de los acuerdos de paz (ACUERDOS DE PAZ, ESQUIPULAS 1987). Asimismo, en un estudio realizado por Lozano et. al (2015, p.40) se señalan varios casos concretos sobre el impacto de los fenómenos naturales en la vida de las poblaciones centroamericanas más pobres.

El primero de ellos Guatemala en los últimos 10 años y el impacto del Huracán Mitch, las tormentas tropicales Stan, Agatha y E12, impactando en la seguridad alimentaria y con ello provocando problemas de desnutrición crónica en niños (as) menores de cinco años aunado a las pérdidas de semillas, cosechas y reservas de 
alimentos, obligando así a migraciones forzosas a la población. Por otra parte, se menciona la respuesta de las comunidades de Waslala y El Cuá en Nicaragua, casos en que "la mayoría de los agricultores que cultivan para el autoconsumo con poca producción para la venta, al perder sus cultivos por un evento climático extremo, básicamente pierden su subsistencia las personas afectadas en la mayoría de los casos migran a verse en esta situación (CEPEDA Y VIGNOLA 2011 citado por LOZANO et. al 2015, p.40).

A nivel de la integración regional centroamericana, la nueva Estrategia Regional Ambiental Marco 2015-2020 (2015) reconoce y señala la importancia de las acciones de mitigación y prevención en la medida que la vulnerabilidad climática incide sobre la estabilidad socioeconómica regional:

La recurrencia de sequías, inundaciones y huracanes pone en riesgo las economías de la región basadas en recursos naturales que dependen fuertemente del clima (agricultura, turismo, etc.).

En este contexto los países de la región han demostrado el interés de encaminar acciones frente a la amenaza del cambio climático, redoblando esfuerzos en este sentido, ya que por un lado tienen que atacar las causas estructurales de la vulnerabilidad socioeconómica y ambiental para mejorar la capacidad de adaptación de als poblaciones y por otro reducir sus emisiones de $\mathrm{CO} 2$, Estrategia Regional Ambiental Marco 2015-2020 (2015, p.9).

Esta es la razón por la que esta nueva estrategia se ha propuesto "transversalizar el enfoque de mitigación y adaptación al cambio climático y la gestión integral del riesgo en todas las políticas y planes nacionales para asegurar la protección de la vida de la población de la región y de sus bienes privados y públicos" (Estrategia Regional Ambiental Marco 2015-2020 (2015 p.13). 


\section{Consecuencias del Cambio Climático en las Poblaciones Indígenas}

Tal y como la Unión Internacional para la Conservación de la Naturaleza (UICN) declaraba en su informe sobre pueblos indígenas y tradicionales y cambio climático, en abril de 2008, es de amplia aceptación el hecho de que "las comunidades pobres que dependen de los recursos naturales en el mundo en desarrollo son particularmente vulnerables al cambio climático" (UICN 2008, p.1).

Este informe coincidía con otros documentos relacionados, como el Informe Stern de 2006 y el Cuarto Informe de Evaluación del Grupo Intergubernamental de Expertos sobre el Cambio Climático (PICC) de 2007, en los que se afirmaba que "quienes mayormente sufrirán las consecuencias del cambio climático serán las comunidades más pobres y vulnerables del mundo, incluyendo los pueblos indígenas y tradicionales" (Ibíd. 2).

Debido a la convergencia de vulnerabilidades biofísicas -como la ubicación habitual de sus residencias y la disponibilidad de recursos naturales-, y sociales -como la pobreza, las condiciones de salud y nutrición, y las redes sociales y de movilidad(Ibíd. 4), los pueblos indígenas en diversas partes del mundo ya han comenzado a percibir los efectos del cambio climático como una realidad y un problema más allá de lo ambiental, con severas implicaciones socioeconómicas (ONU 2010, p.114). Los cambios en las dinámicas medioambientales provocarían profundas modificaciones en las prácticas ancestrales y en los modos de producción, en aspectos que han sido identificados por el Foro Permanente para las Cuestiones Indígenas de Naciones Unidas, tal y como se detalla a continuación: 
Tabla 4: Aspectos afectados y potencialmente afectados por los efectos del cambio climático Fuente: Elaboración propia con base en ONU.

\begin{tabular}{|c|c|}
\hline $\begin{array}{l}\text { Aspectos ya afetados por efectos del } \\
\text { cambio climático }\end{array}$ & $\begin{array}{l}\text { Aspectos potencialmente afectados } \\
\text { por efectos del cambio climático }\end{array}$ \\
\hline $\begin{array}{l}\text { Patrones migratorios } \\
\text { de peces, } \\
\text { aves y mamíferos }\end{array}$ & $\begin{array}{l}\text { La caza, la pesca } \\
\text { y las prácticas de pastoreo de los } \\
\text { pueblos indígenas }\end{array}$ \\
\hline $\begin{array}{c}\text { El calendario y los ciclos de floración, la } \\
\text { migración } \\
\text { y aparición de insectos }\end{array}$ & $\begin{array}{l}\text { La caza, la pesca } \\
\text { y las prácticas de pastoreo de los } \\
\text { pueblos indígenas }\end{array}$ \\
\hline $\begin{array}{c}\text { El calendario y los ciclos de floración, la } \\
\text { migración } \\
\text { y aparición de insectos }\end{array}$ & $\begin{array}{l}\text { El sustento de los pastores. Las } \\
\text { actividade agrícolas tradicionales de los } \\
\text { pueblos indígenas habitantes de } \\
\text { regiones monatañosas. }\end{array}$ \\
\hline $\begin{array}{c}\text { El tamaño de las poblaciones } \\
\text { de ciertas plantas } \\
\text { y animales }\end{array}$ & $\begin{array}{c}\text { Las prácticas culturales y rituales (no sólo } \\
\text { los relacionados con determinadas } \\
\text { especies o ciclos anuales, sino también } \\
\text { con lugares específicos y sitios } \\
\text { espirituales) }\end{array}$ \\
\hline $\begin{array}{l}\text { La disponibilidad de recursos } \\
\text { hídricos }\end{array}$ & La salud de las comunidades Indígenas \\
\hline $\begin{array}{l}\text { La disponibilidade de áreas de pastoreo } \\
\text { y el tamaño de los cultivos }\end{array}$ & $\begin{array}{l}\text { Los ingresos } \\
\text { producto del turismo. }\end{array}$ \\
\hline
\end{tabular}

Los aspectos ubicados en la columna de la derecha, revelan no solamente la potencial modificación de prácticas sociales ancestrales, sino además la potencial incidencia de los efectos del cambio climático en condiciones socioeconómicas y sociodemográficas.

En el caso centroamericano la realidad no es distinta. Representantes de organizaciones indígenas como Sotz'il -en el marco del Foro Regional Estrategia de Agro/ambiente y Salud del Sistema de Integración Centroamericana (SICA)-, han externado su preocupación por los efectos ya percibidos en la región: 
la manifestación de estos efectos, que ya son catastróficos, se perciben fácilmente cuando enfrentamos en nuestros territorios, desórdenes climáticos: sequías extensas, pérdidas en la agricultura y seguridad alimentaria, lluvias intensas (inundaciones, deslaves), pérdida de ecosistemas, efectos en las actividades productivas y la seguridad alimentaria de los pueblos indígenas, efectos en la salud de los pueblos indígenas y pérdida de identidad y cultura (BATZIN, 2011).

Vale resaltar que uno de los efectos que más preocupan es la inminente afectación de la producción agrícola de cultivos como el maíz, el frijol, la yuca y el plátano, base de la tradicional alimentación de los pueblos indígenas mesoamericanos.

Uno de los riesgos latentes es precisamente que esta afectación tenga incidencia directa sobre las problemáticas de hambre, desnutrición y malnutrición en la región (Ibídem.).

La amenaza del cambio climático en la población indígena centroamericana: reflexiones finales

Resulta crucial, en el marco de la identificación de las vulnerabilidades de los pueblos indígenas centroamericanos, una aproximación a la realidad sociodemográfica de estos grupos a partir de información actualizada y fidedigna, que resulte adecuada para el mapeo de las vulnerabilidades de poblaciones que trascienden los límites del Estado-nación. En el caso concreto del impacto del cambio climático se constituye ya como una realidad que afecta de manera particular a los pueblos indígenas como poblaciones en riesgo.

Los efectos resultan alarmantes no solo en términos de las modificaciones medioambientales sino además en términos de sus consecuencias socioeconómicas y sociodemográficas.

En términos de políticas, el cambio climático se ha reconocido en las estrategias ambientales implementadas en el marco del sistema de integración regional como un factor que incide en los desplazamientos internos y externos, junto con elementos económicos, familiares y de seguridad. Así, surge la necesidad de adecuar las políticas de mitigación y prevención a las necesidades y realidades de los grupos indígenas centroamericanos y a su vez, ajustar los marcos jurídicos en términos 
humanitarios para hacerle frente a las posibles aleadas migratorias que podrían generarse en el istmo producto de un fenómeno natural extremo.

Tabla 5: Los derechos indígenas territoriales en América Central según constitución política vigente Fuente: Elaboración propia a partir de C.Barié 2003.

\begin{tabular}{lcl}
\hline País & Año de la constitución & Derechos indígenas territoriales \\
\hline Belice & 1981 & No figuran. \\
\hline Costa Rica & 1949 & No figuran. \\
\hline El Salvador & 1983 & Arts. 103,105 y 114. \\
\hline Guatemala & 1986 & Arts. $62,67,68,121$ y 142. \\
\hline Honduras & 1982 & Arts. $173,300,346$ y 354. \\
\hline Nicaragua & 1987 & Arts. $5,89,107,180$ y 181. \\
\hline Panamá & 1972 & Arts. $77,122,123$ y 254. \\
\hline
\end{tabular}

\section{Referências}

ALBO, A. Y ORDAZ, J. 2011. Migración y cambio climático: El caso mexicano. Disponible en: <https://www.bbvaresearch.com/KETD/fbin/mult/WP_1127_Mexico_tcm346-

267325.pdf?ts=1410>, 2011.

BARIÉ, CLETUS. 2003. Pueblos Indígenas y Derechos Constitucionales: un panorama. Ecuador: Editorial Abya Yala. 2003.

BATZIN, Ramiro. 2011. Los Pueblos Indígenas, el Cambio Climático y la Seguridad Alimentaria [diapositivas]. Disponible en:

<http://www.sica.int/consulta/documento.aspx?idn=59379\&idm=1 > Revisado: 7 de agosto de 2015.

BORRÁS, Susana.. Aproximación al concepto de refugiado ambiental: origen y regulación jurídica internacional (Conferencia impartida en el "III Seminario sobre los agentes de la cooperación al desarrollo: refugiados ambientales, refugiados invisibles?". España: Universidad de Cádiz, 2008.

CÁCERES, Verónica. Los refugiados ambientales: un drama en crecimiento frente a la expansión del capital sobre la naturaleza (Ponencia presentada en las "XXII Jornadas de Historia Económica"). Argentina: Asociación Argentina de Historia Económica y Universidad Nacional de Río Cuarto, 2010. 
CASASFRANCO R. , M. 2002. Las migraciones y los desplazamientos forzados. Retos en Centroamérica y Colombia. Un análisis comparativo e integral desde un enfoque de derechos humanos. Costa Rica: Impresora Obando, 2002.

CEPAL. 2012. La economía del cambio climático en Centroamérica. Disponible en: <http://www.cepal.org/mexico/cambioclimatico/documentos/sintesis_2012baja.pdf>, 2012.

COMISIÓN CENTROAMERICANA DE AMBIENTE Y DESARROLLO. 2014. Estrategia Regional Ambiental Marco 2015-2020. Disponible en:

$<$ https://www.cbd.int/doc/meetings/mar/mcbem-2015-01/other/mcbem-2015-01-estrategiaregional-ccad-es.pdf>, 2014.

COMISIÓN ESPAÑOLA DE AYUDA AL REFUGIADO. 2013. Maras en Centroamérica y México. Disponible en: <http://cear.es/wp-content/uploads/2013/10/CENTROAMERICA.2013.-Maras.pdf> Revisado: 01/09/2014.

DIRECCIÓN GENERAL DE ESTADÍSTICA Y CENSOS DE EL SALVADOR (DIGESTYC). 2007. Censo de Población y Vivienda 2007: Población Indígena. Disponible en:

$<$ http://www.digestyc.gob.sv/index.php/temas/des/poblacion-y-estadisticas-

demograficas/censo-de-poblacion-y-vivienda/poblacion-

censos.html?download=204\%3Apoblacion-indigena> Revisado: 5 de agosto de 2015.

EGENHOFF, T. y STEIN, E. (2011). Seguridad y crimen organizado transnacional. Disponible en: <http://www.kas.de/wf/doc/kas_22430-1522-4-30.pdf?110519174059>. Revisado: 01/09/2014.

FERRIS, E. 2015. Who Will Welcome Climate Change Migrants? Disponible en: <http://ergosum.uaemex.mx/pdfs/pdf_vol_22_1/7_deicy_lozano.pdf?id=10432355002>, 2015.

FRITZ, C. 2010. Climate Change and Migration: Sorting through Complex Issues without the Hype. Migration Policy Institute. Disponible en:

$<\mathrm{http}: / /$ www.migrationpolicy.org/article/climate-change-and-migration-sorting-throughcomplex-issues-without-hype>

<http://www.un.org/esa/socdev/unpfii/documents/SOWIP/en/SOWIP_chapter3.pdf> Revisado: 6 de agosto de 2015.

INSTITUTO NACIONAL DE ESTADÍSTICA GUATEMALA (INE). 2002. Censo Nacional de Población. Disponible en:

<http://www.ine.gob.gt/sistema/uploads/2014/02/20/jZqeGe1H9WdUDngYXkWt3GlhUUQCu kcg.pdf> Revisado: 5 de agosto de 2015.

INSTITUTO NACIONAL DE ESTADÍSTICA Y CENSO DE PANAMÁ. Censos de Población y Vivienda de 2010: Diagnóstico de la Población Indígena de Panamá. Disponible en: <http://www.contraloria.gob.pa/inec/archivos/P6571INDIGENA_FINAL_FINAL.pdf> Revisado: 6 de agosto de 2015. 
INSTITUTO NACIONAL DE ESTADÍSTICA Y CENSOS DE COSTA RICA (INEC). 2011. Resultados generales Censo 2011. Disponible en:

<http://www.inec.go.cr/anda4/index.php/catalog/113/download/713> Revisado: 5 de agosto de 2015.

INSTITUTO NACIONAL DE INFORMACIÓN PARA EL DESARROLLO DE NICARAGUA (INIDE). 2005. VIII Censo de Población y IV de Vivienda 2005, Resumen. Disponible en: $<$ http://www.inide.gob.ni/censos2005/ResumenCensal/Resumen2.pdf> Revisado: 6 de agosto de 2015.

INTERNATIONAL ORGANIZATION FOR MIGRATION [IOM-OIM]. 2012. Migration, climate and the environment. Recuperado de: <http://www.iom.int/jahia/Jahia/definitionalissues>, 2012.

KANDEL, W. (2014). Unaccompanied Alien Children: Potential Factors Contributing to recent Inmigration. Disponible en: <http://fas.org/sgp/crs/homesec/R43628.pdf> Revisado: 01/09/2014.

LOZANO, D. et.al. 2015. "Eventos climáticos extremos y migración interna en

Guatemala, un análisis basado en percepciones de expertos". En: Ciencia ergo-sum. Disponible en:

<http://ergosum.uaemex.mx/pdfs/pdf_vol_22_1/7_deicy_lozano.pdf?id=10432355002>

MÉNDEZ, Rafael (08/12/2008). “25 millones de parias climáticos”, en: El País. España. Disponible en:

<http://www.elpais.com/articulo/sociedad/25/millones/parias/climaticos/elpepusoc/20081208e Ipepisoc_3/Tes>, 2008.

MELÉNDEZ, J. (2014). “Centroamérica, muy vulnerable al cambio climático”. En: EI País. Disponible en:

<http://sociedad.elpais.com/sociedad/2014/06/09/actualidad/1402324929_244265.html>. Revisado: 01/09/2014.

MONTOBBIO, M. (2012). Esquipulas II +25: Centroamérica en perspectiva. Disponible en: $<\mathrm{http}$ ://www.cidob.org/es/publicaciones/notes_internacionals/n1_61/esquipulas_ii_25_centro america_en_perspectiva>. Revisado: 01/09/2014.

NETZAHUALCOYOTZI, R. y FURLONG, A..2013. "Migración centroamericana y globalización”. En: Mesoamérica: Integración regional y geopolítica (editado por Willy Soto Acosta). Costa Rica: Escuela de Relaciones Internacionales de la Universidad Nacional, 2013.

ONU. 2010. State of the World's Indigenous People Chapter 3: Environment. Disponible en: <http://www.un.org/esa/socdev/unpfii/documents/SOWIP/en/SOWIP_web.pdf>, 2010.

Organización Internacional del Trabajo. Convenio 169 sobre Pueblos Indígenas y Tribales en Países Independientes. Disponible en:

<http://www.ilo.org/indigenous/Conventions/no169/lang--en/index.htm>. Revisado: 5 de agosto de 2015. 
OROZCO, M. y YANSURA, J. (Agosto 2014). Comprender la Migración Centroamericana: La crisis de migrantes menores de edad centroamericanos en contexto. Estados Unidos de América: Diálogo Interamericano, 2014.

PALMIERI, F. (2014). Children Migrating from Central America: creating a Humanitarian Crisis. Disponible en: <http://docs.house.gov/meetings/FA/FA07/20140625/102418/HHRG113-FA07-Wstate-PalmieriF-20140625.pdf> Revisado: 01/09/2014.

PERALES, Arturo (2010). “Refugiados ambientales, cambio climático y capitalismo". En: Alternativas. Revista Electrónica, Año V, Número 68. México: Red Mexicana de Acción frente al Libre Comercio / RMALC, 2010.

PROGRAMA ESTADO DE LA NACIÓN. 2011. Cuarto Informe Estado de la Región en Desarrollo Humano Sostenible, capítulo 2. Costa Rica: Programa Estado de la Nación. Disponible en:

<http://www.estadonacion.or.cr/files/biblioteca_virtual/centroamerica/004/cap02_demografico -REGION004.pdf>. Revisado: 6 de agosto de 2015.

PROGRAMA ESTADO DE LA NACIÓN. 2011. Cuarto Informe Estado de la Región en Desarrollo Humano Sostenible, capítulo 3. Costa Rica: Programa Estado de la Nación. Disponible en:

<http://www.estadonacion.or.cr/files/biblioteca_virtual/centroamerica/004/cap03_equidad_so cial-REGION004.pdf>. Revisado: 6 de agosto de 2015.

RODRÍGUEZ, G. 2013. Estudio: Tendencias migratorias, pautas y marcos legales de migración en América Central. Disponible en: <www.cidehum.org>, 2013.

SOTO-ACOSTA, Willy. 2014. "Cambio climático y desplazados ambientales en la sociedad transnacional”. En: Relaciones Internacionales, N. 87.1. Costa Rica: Escuela de Relaciones Internacionales de la Universidad Nacional.

STATISTICAL INSTITUTE OF BELIZE. 2010. Customized data Census 2010. Disponible en: <http://200.32.211.52:8000/cgibin/RpWebEngine.exe/PortalAction?\&MODE=MAIN\& BASE=BELIZE2010\&MAIN=WebServerMain.inl> Revisado: 5 de agosto de 2015.

UNICEF. (April 2013). International Migration, Children and Adolescents Population Dynamics. Disponible en:

$<$ http://www.unicef.org/socialpolicy/files/Population_Dynamics_and_Migration.pdf> Revisado: 01/09/2014.

UNIDAD COORDINADORA PUEBLOS INDÍGENAS EN AMÉRICA LATINA Y EL CARIBE (KIVLAK/GIZ). 2010. Pueblos Indígenas en Honduras. Disponible en:

<http://www.giz.de/expertise/downloads/giz2010-es-laenderpapier-honduras.pdf> Revisado: 6 de agosto de 2015. 
UNIÓN INTERNACIONAL PARA LA CONSERVACIÓN DE LA NATURALEZA (UICN). 2008. Los pueblos indígenas y tradicionales y el cambio climático, 2008.

UNITED NATIONS PERMANENT Forum on Indigenous Issues. State of the World's Indigenous People, chapter 3: Environment., 2010.

' Hace referencia al Nicaraguan Adjustment and Central American Relief Act.

ii El impacto del Huracán Mitch en la región produjo un desplazamiento del 22465 hondureños y una oleada migratoria de nicaragüenses hacia Costa Rica. Mientras que el terremoto en El Salvador (2001) incidió también en el desplazamiento de personas (CASASFRANCO, 2002, p. 119). 